\section{The early diagnostic and prognostic values of blood lactate concentrations in Japanese monkeys with acute gastric dilatation}

\section{Tohru Kimura}

Center for Experimental Animals, National Institutes of Natural Sciences, Myodaiji, Okazaki, Aichi, Japan

\section{Abstract}

The purpose of this study is to determine the relationship between blood lactate and pyruvate concentrations in Japanese monkeys. On the basis of these results, I investigated whether measurement of blood lactate concentrations in ill animals with acute gastric dilatation was useful in identifying an earlier diagnosis and prognosis. Blood lactate and pyruvate concentrations were determined in 43 Japanese monkeys. Using a portable lactate analyzer, blood lactate concentrations in 4 ill animals were examined at the on set of acute gastric dilatation and after the recovery from this disease. The present results revealed that blood lactate concentrations linearly correlated with pyruvate concentrations in Japanese monkeys: $\quad \mathrm{y}=0.7214+0.0142 \mathrm{x}, \quad \mathrm{r}=0.7813$ $(\mathrm{P}<0.01)$. At the onset of acute gastric dilatation, the monkeys showed high lactate concentrations. Blood lactate levels in these ill animals remained elevated under uneventful circumstances. After emergency treatment, their lactate concentrations apparently decreased to the reference range $(\mathrm{P}<0.05)$. It was probable that persistent mechanical compression by the expanded stomach on the portal vein and/or caudal vena cava obstructed the blood flow, resulting in persistent hyperlactic acidemia from anaerobic metabolism. I concluded that blood lactate concentrations could serve as a reliable diagnostic predictor of clinical out come in Japanese monkeys suffering from acute gastric dilatation. Monkeys with remarkable post-treatment decreases in blood lactate concentrations showed an excellent improvement in survival.

\section{Introduction}

Acute gastric dilatation has been reported in human beings, dogs (large breeds with a deep chest), horses, ruminants, pigs, cats, foxes, mink, rabbits, nutria, guinea pigs, rats, mice and captive wild carnivores in addition to laboratory-housed nonhuman primates. ${ }^{1}$ Acute gastric dilatation is a life-threatening condition manifested by gross distention of the stomach with abundant fluid and/or gas. It has been postulated that acute gastric dilatation is multifactorial in origin in all nonhuman primates. These factors contain psychological or behavioral stress, food restriction, overeating, fermentable substrate, fermentable microflora, prior anesthesia and abnormal gastric function. ${ }^{2}$ The exact etiology and pathophysiology of this disease, however, still remain uncertain. Acute gastric dilatation in laboratory monkeys is widely assumed to be precipitated by alterations in daily husbandry program. ${ }^{3-5}$ And this disease is often related to overeating which results in the production of Clostridium perfringens type $\mathrm{D}$ toxin derived from a commercial primate diet.2,4,6-8 In a previous report, the investigators attempted to inoculate directly healthy monkeys with gastric contents of monkeys that died of acute gastric dilatation. ${ }^{9}$ However, this trial also failed to reproduce the same ill conditions in these animals.

In human medicine, blood lactate and pyruvate concentrations are used clinically as a prognostic indicator for critical ill patients. ${ }^{10-12}$ Recently, a portable lactate analyzer that is capable of measuring in a small volume sample has been validated for use in dogs. 13,14 This analyzer provides a convenient means of whole-body screening in the management of critical diseases, as compared with the measurement of blood pyruvate levels. In veterinary medicine, blood lactate concentrations are thus employed as a predictor of gastric necrosis and survival among dogs with gastric dilatation-volvulus. ${ }^{15-17}$

The purpose of this study was to determine the relationship between blood lactate and pyruvate concentrations in Japanese monkeys. On the basis of these results, I investigated whether measurement of blood lactate concentrations in response to treatment for ill animals with this disease was useful in identifying an earlier diagnosis and more accurate prognosis.

\section{Materials and Methods}

\section{Animals}

We used 43 Japanese monkeys (Macaca fuscata, 20 male and 23 female, 3.00 to $8.55 \mathrm{~kg}$ ) in this study. The estimated age range of the monkeys was 3 to $6 \mathrm{y}$ at the time of introduction and they were used for this study 2 months after acclimatization. These animals were purchased from a commercial supplier (Hamri Co., Ltd., Ibaraki, Japan). Before the experiment, the monkeys were determined to be healthy on the basis of general appearance, activity, and tuberculosis skin testing. All ani-
Correspondence: Tohru Kimura, Center for Experimental Animals, National Institutes of Natural Sciences, 38, Nishigo-naka, Myodaiji, Okazaki, Aichi, 444-8585, Japan.

Tel. +81.564.557.882 - Fax: +81.564.557.881

E-mail: kimura@nips.ac.jp

Key words: acute gastric dilatation, diagnosis, Japanese monkeys, lactate, prognosis, pyruvate.

Received for publication: 3 November 2013.

Revision received: 4 December 2013.

Accepted for publication: 19 December 2013.

This work is licensed under a Creative Commons Attribution NonCommercial 3.0 License (CC BYNC 3.0).

CC Copyright T. Kimura, 2014

Licensee PAGEPress srl, Italy

Veterinary Science Development 2014; 4:5155

doi:10.4081/vsd.2014.5155

mals were seronegative for B virus by enzymelinked immunosorbent assay. The monkeys were housed individually in stainless steel cages $(60 \times 70 \times 160 \mathrm{~cm})$ in an animal room controlled at $25 \pm 1^{\circ} \mathrm{C}$ and $50 \pm 10 \%$ relative humidity with 10 to 15 exchanges of $100 \%$ fresh air hourly and a 12:12-h light:dark cycle (lights on, 0600). They were fed a commercial primate food (PS, Oriental Yeast Co., Ltd., Tokyo, Japan), provided ad libitum and supplemented with a variety of fresh fruit, vegetables, and other treats daily. Water was provided through an automatic watering system furnished to each cage. All procedures involving animals were approved by the Animal Use Committee of National Institutes of Natural Sciences of Japan and followed institutional guidelines of animal care and experimentation.

\section{Blood sample collection}

Blood samples $(5 \mathrm{~mL})$ were collected without anticoagulant from the cephalic vein of each animal. At $30 \mathrm{~min}$ after collection of blood samples, sera were separated by centrifugation at $1500 \times \mathrm{g}$ for $10 \mathrm{~min}$ for biochemical analysis.

Blood lactate concentrations were determined with the use of the lactate oxidase-peroxidase method (Determiner LA, Kyowa medex Co., Ltd. Tokyo, Japan). Blood pyruvate concentrations were measured by pyruvate oxidase-peroxidase method (Determiner PA, Kyowa medex Co., Ltd. Tokyo, Japan).

\section{Statistical evaluation}

Data are expressed as mean \pm 1 standard deviation (SD); a linear regression study was performed to investigate the correlation between the lactate and pyruvate concentrations. (Statcel, OMC Ltd., Tokorozawa, Japan). 


\section{Four monkeys with naturally occur- ring acute gastric dilatation}

Electrochemical assay system with singleuse electrode strip for measuring lactate in whole body.

On the basis of reference values of the lactate concentrations in 43 Japanese monkeys, electrochemical assay system with single-use electrode strip was applied to measurements of lactate concentrations in whole body of simian patients. Four ill animals (Japanese monkeys, 3 male and 1 female) with naturally occurring acute gastric dilatation were investigated for measuring their lactate concentrations via the electrochemical assay system. ${ }^{18}$ Lactate concentrations of these monkeys were examined on the onset of acute gastric dilatation and after the complete recovery from this disease. A small amount of blood samples $(0.5 \mathrm{~mL})$ were collected without anticoagulant from the cephalic vein of each animal. An electrode strip was inserted into the strip inlet of the blood lactate test meter (Lactate Pro, Arkray, Inc., Kyoto, Japan), and the meter switched on automatically. After the other end of the strip touched a drop of whole blood, $5 \mu \mathrm{L}$ of whole blood was aspirated and measurement started. The lactate concentration was displayed 60 seconds after beginning of this measurement.

Data are expressed as mean $\pm 1 \mathrm{SD}$; statistical analysis was performed using a paired ttest (Statcel, OMC Ltd., Tokorozawa, Japan). Blood lactate concentrations after emergency treatment were compared with those at the onset of this disease.

\section{Results}

\section{The relationship between blood lactate and pyruvate concentra- tions}

The relationship between the lactate and pyruvate concentrations in Japanese monkeys is shown in Figure 1. The reference range for the blood lactate concentrations was 0.67 to $4.43 \mathrm{mmol} / \mathrm{L}(2.08 \pm 0.98 \mathrm{mmol} / \mathrm{L}$, mean $\pm \mathrm{SD})$. The measurements of the blood pyruvate concentrations were 35.22 to $267 \mu \mathrm{mol} / \mathrm{L}$ $(96.24 \pm 53.94 \mu \mathrm{mol} / \mathrm{L})$. The equation for the regression line was as follows: $\mathrm{y}=0.7214+0.0142 \mathrm{x}$. The correlation coefficient (r) between lactate concentrations and pyruvate concentrations measured from venous blood samples was $0.7813(\mathrm{P}<0.01)$. In the present results, lactate levels increased proportionately with increasing pyruvate concentrations. This metabolic action was accompanied by an increase in lactate and pyruvate concentrations that maintained a constant ratio between both parameters.

\section{Clinical findings}

The clinical appearance of acute gastric dilatation in a Japanese monkey is shown in Figure 2. The first sign of this disorder was occasional mild discomfort and the monkeys refused food. Their face turned pale, and then uncomfortable symptoms reduced their activities. Subsequently, the animals assumed a crouched position or fell prone on the bottom of the cage with their legs pulled up under the body. The ill animals did not suffered from intractable nausea or vomiting and ingesta and blood were not found around the nostrils and mouth. Their markedly distended abdomens had hollow tympanic sounds on percussion, the skin was cold and the oral mucous membranes were cyanotic. The dilated stomach contained ingesta, water and varying quantities of gas.

Affected animals have been overfed prior to the onset of acute gastric dilatation. One animal that was overfed was on a restricted diet intake as part of the experiment and was subsequently allowed to overeat late at night. The other animal had received an anesthetic, immobilizing or tranquilizing drug before suffering from this disease, and then this monkey was overfed with expanded pellets soon after recovery from anesthesia.

\section{Therapy}

Even though the monkeys clinically showed no signs of acute gastric dilatation, there were high lactate concentrations ( $>4.44 \mathrm{mmol} / \mathrm{L}$ ) under uneventful circumstances.

Immediately after monkeys were suspected of suffering from acute gastric dilatation, ketamine hydrochloride (2.5 $\mathrm{mg} / \mathrm{kg}$, i.m.) was administrated with medetomidine hydrochloride $(60 \mu \mathrm{g} / \mathrm{kg}$, i.m.) and midazolam $(0.3$ $\mathrm{mg} / \mathrm{kg}$, i.m.). The first therapy consisted of emptying and lavage of the stomach. The gastric contents were evacuated via the stomach tube. The stomach of the monkey was distributed with semi-liquid, gaseous ingesta which had the fetid order of fermenting mash. The high intragastric pressure was relieved by gastric intubation which allowed both gas and fluid to escape. All the monkeys survived and their symptoms completely disappeared following the evacuation of the stomach contents. Their distressing symptoms were attributed to the circulatory and respiratory embarrassment occurring subsequent to gastric dilatation. Antibiotics (enrofloxacin, $20 \mathrm{mg} / \mathrm{kg}$, i.m.; Baytril, Bayer Japan Co., Ltd., Tokyo, Japan) and steroids [prednisolone, $0.25 \mathrm{mg} / \mathrm{kg}$, s.c.; Prednisolone injection (Fuijita), Fujita Co., Ltd., Tokyo, Japan] were given to treat bacteremia and endotoxic shock.

After treatment of acute gastric dilatation, affected monkeys began to be fed with fruits such as one half bananas and one fourth apple. In addition, probiotics (Streptococcus faecalis
T-110, Clostridium butyricum T0-A, Bacillus mesentericus T0-A, Bio-three, Toa Pharmaceutical Co. Ltd., Tokyo, Japan) and digestive enzymes (diastase, Nipro Pharm Co. Ltd., Osaka, Japan) were administrated to the affected monkeys. Feeding smaller meals several times daily reduced recurrence rate of this disease.

The changes in blood lactate concentrations of the treated monkeys are shown in Figure 3. The portable lactate analyzer was available to obtain quickly blood lactate concentrations. At the onset of acute gastric dilatation, the monkeys showed high lactate concentrations (6.66$13.32 \mathrm{mmol} / \mathrm{L}, 11.21 \pm 4.23 \mathrm{mmol} / \mathrm{L})$, as compared with our reference values. These animals developed lactate acidosis in association with metabolic acidosis. After emergency treatment, their lactate concentrations apparently decreased to $1.11-3.33 \mathrm{mmol} / \mathrm{L}(2.13 \pm 0.93$ $\mathrm{mg} / \mathrm{dL}$ ), reaching the normal range. There was a significant difference in lactate concentrations between the onset and post-treatment measurement $(\mathrm{P}<0.05)$. Four ill monkeys whose lactate concentrations began to return to the reference range showed a better clinical course following emergency treatment (evacuation of the gas and liquid contents and gastric decompression). The ill animals with decreased blood lactate concentrations had an excellent post-treatment recovery from acute gastric dilatation.

\section{Discussion}

This clinical study reported here was tried to determine the relationship between blood lactate and pyruvate concentrations in Japanese monkeys. In addition, I investigated whether measurement of blood lactate concentrations in response to emergency treatment for affected monkeys with this disease assists in identifying an earlier and more accurate prognosis.

The present results demonstrated that the changes in lactate concentrations were significantly correlated with increased pyruvate concentrations in Japanese monkeys $(\mathrm{P}<0.01)$. Under normal conditions of aerobic metabolism, lactate is produced in low concentrations. From the previous studies, the normal ranges of lactate concentrations are as follows: dogs $(<2.0-6.5 \mathrm{mmol} / \mathrm{L})$, cats $(<2.0 \mathrm{mmol} / \mathrm{L})^{19}$ and rhesus macaques $(<2.0 \mathrm{mmol} / \mathrm{L}) .{ }^{20}$ The present data obtained from Japanese monkeys agreed with these reference values in veterinary medicine. These results revealed that blood lactate concentrations linearly correlated with pyruvate concentrations within double the reference ranges. It was probable that almost all lactate was derived from pyruvate, which was generated as a normal step in glycolysis. It takes us several hours to assess 
accurately blood pyruvate levels. These findings provided evidence that blood lactate concentrations was a useful parameter in prompt diagnosis and prediction of simian acute gastric dilatation.

Elevated blood lactate concentrations are most commonly a result of systemic hypoperfusion and tissue hypoxia that develop as a course of shock. ${ }^{21,22}$ During anaerobic conditions, pyruvate is converted to lactate for energy [adenosine triphosphate (ATP) production]. In more advanced stages of this condition, lactate production exceeds the rate of metabolism. 15

In the human definitions, hyperlactacidemia is usually a mild to moderate increase $(2-5 \mathrm{mmol} / \mathrm{L})$ in lactate concentrations without concurrent metabolic acidosis. Lactic acidosis shows a persistently elevated lactate concentration (often $>5 \mathrm{mmol} / \mathrm{L}$ ) in association with metabolic acidosis $(\mathrm{pH}<7.35) .{ }^{19}$ A precise definition of lactic acidosis has not yet been determined in veterinary medicine. In the dogs with gastric dilatation and volvulus, ill animals suffering from plasma lactate levels $>6 \mathrm{mmol} / \mathrm{L}$ had a $58 \%$ survival rate, whereas those with values $<6$ $\mathrm{mmol} / \mathrm{L}$ had a survival rate of $99 \% .15$ Although the definition of hyperlactacidemia in monkeys is not known, blood lactate levels in 4 affected monkeys remained elevated above the present reference values $(>4.44 \mathrm{mmol} / \mathrm{L})$.

The prognosis of acute gastric dilatation must be guarded in affected monkeys, since the changes of the other parameters are not always reflected by the clinical manifestations. The pathogenesis of this disease involves a more complex progression of physiological disturbances that can rapidly (within a few minutes) culminate in death unless immediate medical and surgical treatment is provided.

Recently, hand-held lactate analyzers have become popular and affordable for measuring blood lactate concentrations on a small volume sample in dogs. This apparatus has raised the predictable values of measuring blood lactate concentrations in veterinary medicine. 13,14

I propose the following developmental processes of simian acute gastric dilatation. i) After vigorous restriction of foods and moisture or occasional confinement in a chair during research procedures, gulping down monkey diets and water predisposes experimental monkeys to stomach expansion. ii) A marked increase in gastric $\mathrm{pH}$ allows anaerobic bacteria such as Clostridium perfringens to multiply rapidly, accompanied by the production of their toxins [in the previous assessment of heat resistant bacterial spores from monkey diets, the number of those spores was $1.2 \times 104 / \mathrm{g}-$ $4.6 \times 104 / g$. (unpublished data)]. iii) Large quantities of frothy liquid contents are produced by fermentable substances and the presence of flora capable of fermentation. iv)
Progressive gastric expansion, associated with vascular stasis in the stomach, compress the adjacent organs such as the spleen, liver, diaphragm, portal vein and inferior vena cava. Mechanical obstruction causes insufficient venous circulation and vascular stasis of the gastric wall. v) Following a paralysis of the stomach, decreased muscle tone allows the entrance of exogenous air into the upper gas- trointestinal tract without swallowing. vi) These etiological factors eventually lead to the development of hypotension, hypovolemia, low blood velocity, poor tissue or systemic perfusion, metabolic acidosis, hypoxia and severe shock.

Sitting in a restraint chair during the experiment seemed to cause vascular stasis resembling physiopathology in deep-vein thrombosis

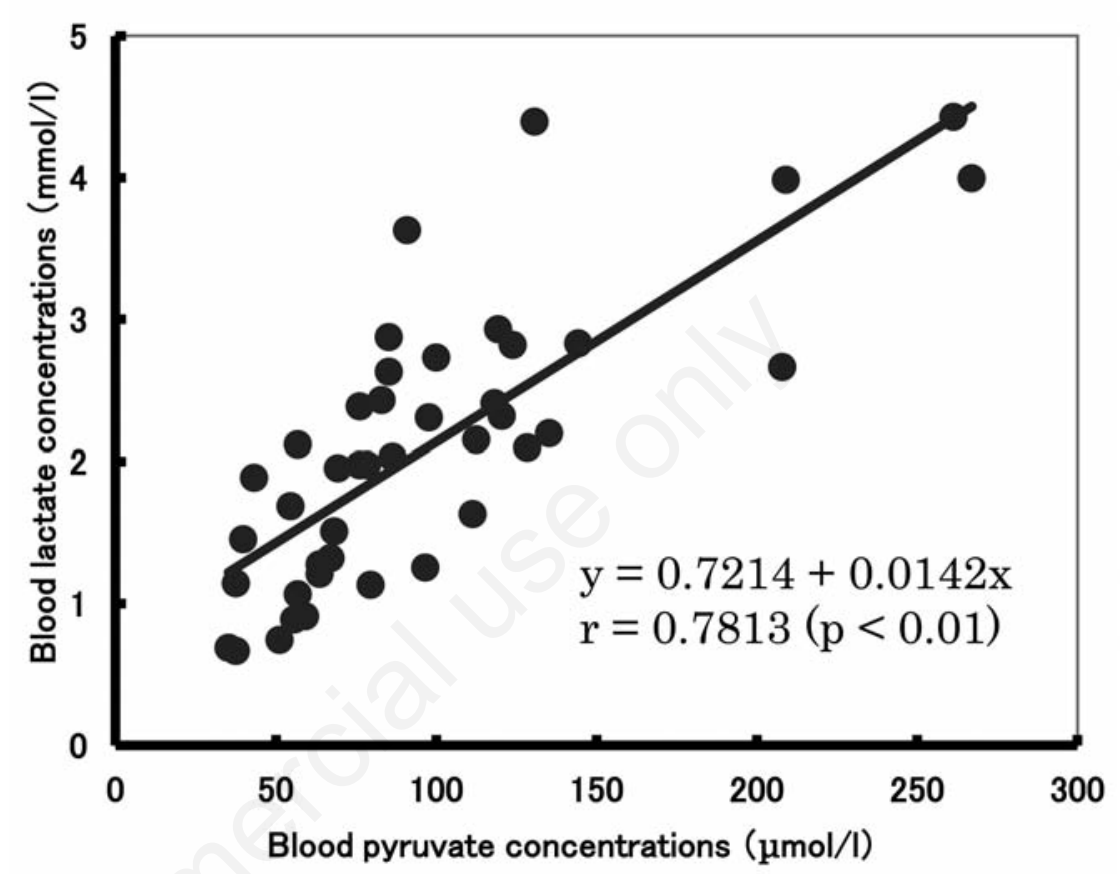

Figure 1. The relationship between the lactate and pyruvate concentrations in Japanese monkeys.

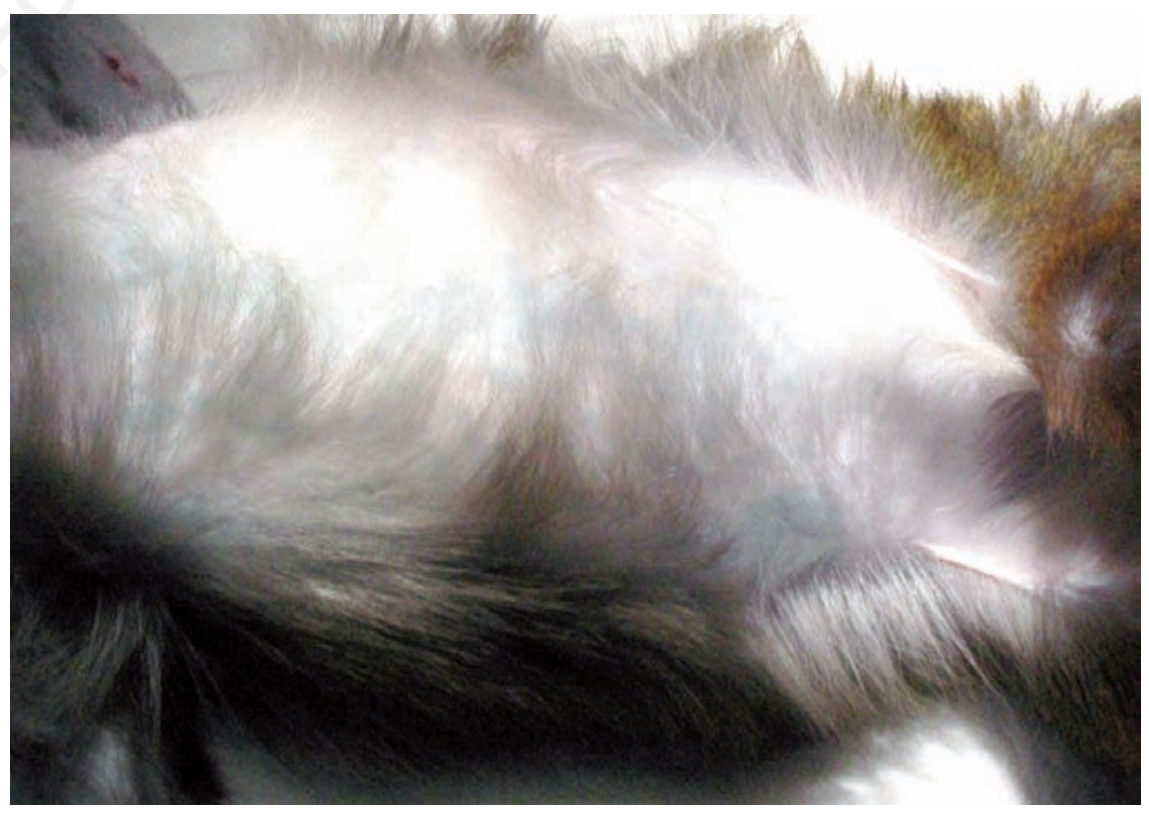

Figure 2. The clinical appearance of acute gastric dilatation in a Japanese monkey. 


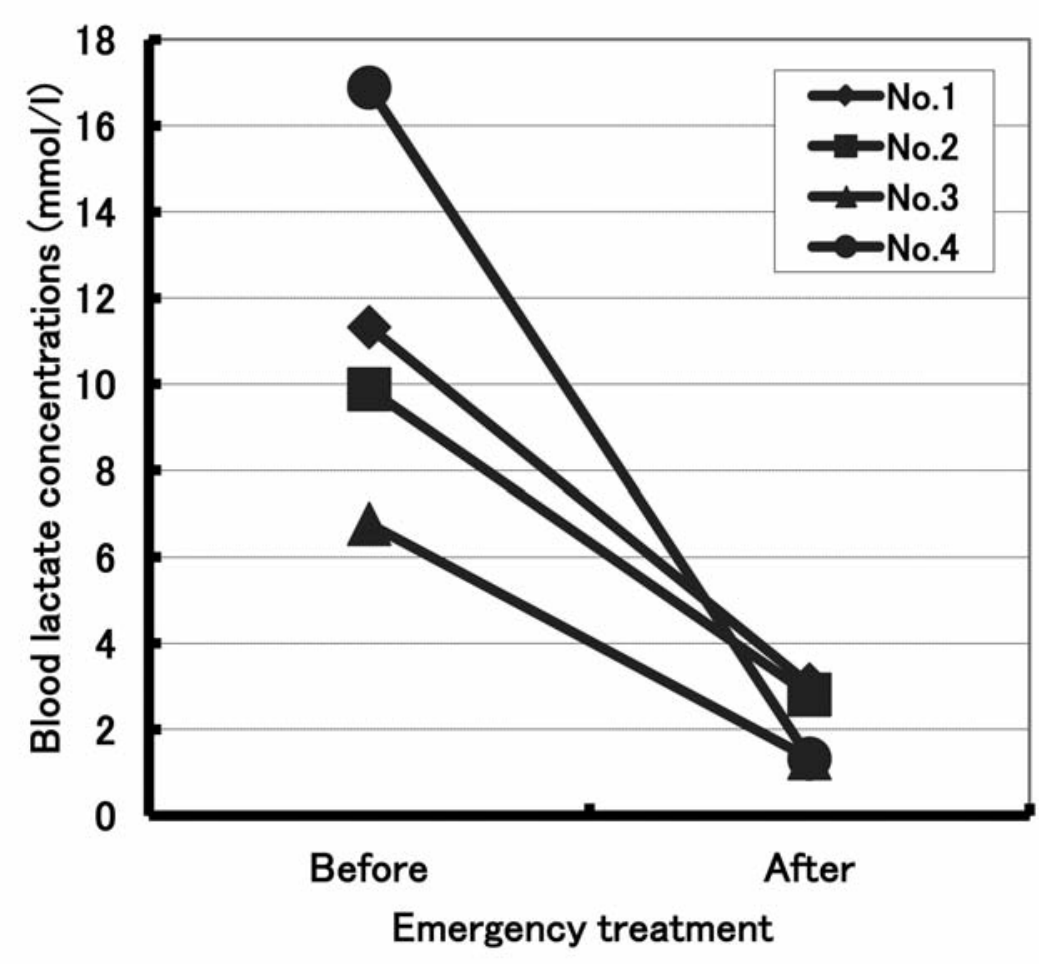

Figure 3. The changes in blood lactate concentrations of 4 treated monkeys (No. 1-4). Note apparent decreases in blood lactate concentrations following emergency treatment. Before: at the onset of acute gastric dilatation. After: 4-7 days after emergency treatment.

(economy class syndrome). In rhesus monkeys with a prior history of acute gastric dilatation, recent radiographic study on gastric motility reported no significant differences from healthy control monkeys, indicating that slow gastric emptying times do not play a role in this syndrome.23 Although abnormalities in gastric motility have been suspected to be deleterious risk factors for acute gastric dilatation, it is unlikely that this disease develops secondary to gastric stasis condition. Persistent mechanical compression by the expanded stomach on the portal vein and/or caudal vena cava obstructed the blood flow, resulting in persistent metabolic acidosis from anaerobic metabolism. In the recent study, blood gas analysis showed that the monkeys suffering from this disorder had severe and persistent metabolic acidosis. In addition, the authors described that regular blood gas analysis permitted follow-up of this disease and obviate excessive administration of sodium bicarbonate. ${ }^{24}$ Hobbs et al. ${ }^{20}$ reported that after resuscitation therapy in rhesus macaques with multiple severe traumas, serum lactate was the only predictor of acidosis in association with survival. Therefore, it is important to determine blood lactate concentrations for early diagnosis of this disease. It is probable that the prognostic values of blood lactate concentrations are attributable to the association with systemic hypoperfusion and/or gastric ischemia following marked stomach expansion.

\section{Conclusions}

I concluded that blood lactate concentrations could serve as a reliable diagnostic predictor of clinical outcome in Japanese monkeys suffering from acute gastric dilatation. Monkeys with remarkable post-treatment decreases in blood lactate concentrations showed an excellent improvement in survival.

\section{References}

1. Van Kruiningen HJ, Gregoire K, Meuten DJ. Acute gastric dilatation: a review of comparative aspects, by species, and a study in dogs and monkeys. J Am Anim Hosp Assoc 1974;10:294-324.

2. Pond CL, Newcomer CE, Anver MR. Acute gastric dilatation in nonhuman primates: review and case studies. Vet Pathol 1982;19 Supp 7:127-33.

3. Boyce L, Miller C. Acute gastric dilatation with herniation in rhesus monkey. Vet Med/Small Anim Clin 1980;75:130-1.

4. Newton WE, Beamer PD, Rhoades HE. Acute bloat syndrome in stumptailed macaques (Macaca arctoides): a report of four cases. Lab Anim Sci 1971;21:193-6.

5. Soave OA. Observations on acute gastric dilatation in nonhuman primates. Lab Anim Sci 1978;28:331-4.

6. Bennett BT, Cuasay L, Welsh TJ, Beluhan FZ, Schofield L. Acute gastric dilatation in monkeys: a microbiologic study of gastric contents, blood and feed. Lab Anim Sci 1980;30:241-4.

7. Chapman WL. Acute gastric dilatation in Macaca mulatta and Macaca speciosa monkeys. Lab Anim Care 1967;17:130-6.

8. Cristie RJ, King RE. Acute gastric dilatation and rupture in Macaca arctoides associated with Clostridium perfringens. J Med Primatol 1981;10:263-4.

9. Smith AW, Casey HW, LaCroix JT, Johnson DK. Acute bloat syndrome (gastric dilatation) in Macaca mulatta. J Am Vet Med Assoc 1969;155:1241-4.

10. Debray FG, Mitchell GA, Allard P, et al. Diagnostic accuracy of blood lactate-topyruvate molar ratio in the differential diagnosis of congenital lactic acidosis. Clin Chem 2007;53:916-21.

11. Jansen TC, van Bommel J, Baker J. Blood lactate monitoring in critically ill patients: a systematic health technology assessment. Crit Care Med 2009;37:287-9.

12. Poggi-Travert F, Martin D, Billette de Villemeur T, et al. Metabolic intermediates in lactic acidosis: compounds, samples and interpretation (Review). J Inherit Metab Dis 1996;19:478-88.

13. Ferasin L, Nguyenba TP. Comparison of canine capillary and jugular venous blood lactate concentrations determined by use of an enzymatic-amperometric bedside system. Am J Vet Res 2008;69:208-11.

14. Stevenson CK, Kidney BA, Duke T, et al. Evaluation of the Accutrend for lactate measurement in dogs. Vet Clin Pathol 2007;36:261-6.

15. de Papp E, Drobatz KJ, Hughes D. Plasma lactate concentration as a predictor of gastric necrosis and survival among dogs with gastric dilatation-volvulus: 102 cases (1995-1998). J Am Vet Med Assoc 1999; 215:49-52.

16. Green TI, Tonozzi CC, Kirby R, Rudloff E. Evaluation of initial plasma lactate values as predictor of gastric necrosis and initial and subsequent plasma lactate values as predictor of survival in dogs with gastricdilatation volvulus: 84 dogs (2003-2007). J Vet Emerg Crit Care 2011;21:36-44.

17. Zacher LA, Berg J, Shaw SP, Kudej RK. Association between outcome and changes in plasma lactate concentration 
during presurgical treatment in dogs with gastric-dilatation volvulus: 64 cases (20022008). J Am Vet Med Assoc 2010;236:892-7.

18. Shimojo N, Naka K, Uenoyama $H$, et al. Electrochemical assay system with singleuse electrode strip for measuring lactate in whole blood. Clin Chem 1993;39:2312-4.

19. Pang DS, Boysen S. Lactate in veterinary critical care: pathophysiology and management. J Am Anim Hosp Assoc 2007;43:2709.

20. Hobbs TR, O'Malley JP, Khouangsathiene
S, Dubay CJ. Comparison of lactase, base excess, bicarbonate, and $\mathrm{pH}$ as predictors of mortality after severe trauma in rhesus macaques (Macaca mulatta). Comp Med 2010;60:233-9.

21. Levin GM, Bonczynski JJ, Ludwig LL, et al. Lactate as a diagnostic test for septic peritoneal effusions in dogs and cats. J Am Anim Hosp Assoc 2004;40:364-71.

22. Stevenson CK, Kidney BA, Duke T, et al. Serial blood lactate concentrations in systemically ill dogs. Vet Clin Pathol
2007:36:234-9.

23. Fonton J W, Cosgrove DJ, Golden JG. Gastrin levels and gastric emptying times in rhesus monkeys with a history of acute gastric dilatation. J Med Primatol 1995;24: 243-5.

24. Camapanile N, Rood PP, Yeh P, et al. Acute gastric dilatation after porcine islet transplantation in a cynomolgus monkey - case history and review of the literature. Xenotransplantation 2007;14:265-70. 